\title{
Distributed Network Management Architectures: A Review
}

\author{
Anish Saini \\ Department of Computer \\ Science \& Engineering \\ Echelon Institute of Technology \\ Faridabad, India
}

\author{
Atul Mishra \\ Department of Computer \\ Engineering \\ YMCA University of Science \& \\ Technology, Faridabad, India
}

\author{
A.K Sharma \\ Department of Computer \\ Engineering \\ YMCA University of Science \& \\ Technology, Faridabad, India
}

\begin{abstract}
Network management system leads to centralized system based on SNMP or CMIP management protocol, which does not solve the problem of scalability and flexibility. So a distributed paradigm is followed to make the system decentralized to perform management functions when network start growing significantly. Several distributed management architectures including mobile agent technology, have been recently proposed to answer the scalability limitations of centralized models and the flexibility problems of different network models. Mobile agents decentralize processing and control thereby reducing the management traffic around the management station and distribute processing load. This paper discusses and analyzes the role of mobile agent and compares the network parameters for different models.
\end{abstract}

\section{Keywords}

Mobile agents, Network Management, Distributed, SNMP, Scalability.

\section{INTRODUCTION}

A Network Management System will typically monitor both hardware and software components of a network. Network Management system utilities typically record data from remote points on a network for central reporting to an administrator. Network management system controls a network to make it more efficient and productive. Network management system has: fault management, accounting management, configuration management, security management and performance management. There are two standard protocols for network management and monitoring. Simple Network Management Protocol (SNMP), which defines a set of instructions that can be used to view and change the settings on any SNMP-compliant device. Another one is RMON, or the Remote Monitoring protocol, which bolts on to SNMP and allows remote collection of network information. SNMP is an asymmetric protocol, operating between a manger and an agent. The agent is the device being managed - all its software has to do is implement a few simple packet types and a generic get-or-set function on its MIB variables. The management station presents the user interface

SNMP V1 operates over UDP (User Datagram Protocol), IP (Internet Protocol), CLNS (OSI Connectionless Network Service), DDP (AppleTalk Datagram-Delivery Protocol) and IPX (Novell Internet Packet Exchange). SNMP v1 uses the authentication mechanism of transmitting a "community string" (for example, a password) in clear text, which is very insecure. SNMP V2 also adds improvements in the area of manager to manager communication. GetBulkRequest has been added to retrieve large data amounts by a single request. Earlier GetNextRequest is used iteratively in order to get a bulk of data. SNMP v2 security system is too complex.[1] SNMP V3 provides better security in the form of authentication via hashing, timestamps, and message confidentiality due to the use of encryption.

Centralized network management systems based on client/server is known by a low degree of scalability and flexibility, and cannot fulfill the needs of telecommunication networks of the current scenarios. SNMP \& CMIP both address the problem of interoperability in heterogeneous environments[2]. These protocols are based on static and centralized client/server model, where every network element sends all the data to a central location. In centralize management system agents monitor the system and collect data, which can be accessed by applications via management protocols (SNMP). SNMP manages and monitors only network elements and agents provide a limited and fixed set of functions [3]. This system is highly dependent on central management station and it is the only spot of failure. Sometimes the management host does not fail, but a fault appears in the networks, which can damage that network because it doesn't have any management capabilities.

Centralize management systems follow a platform-centered model and are not scalable therefore, if the number of devices and managed variables, or speed of the network increases or if management communications rates are restricted, then the system quickly becomes unmanageable.

However, centralize management systems produce too much traffic in network

\section{CLIENT SERVER BASED 3 - TIER MODEL}

Figure 1 shows three tier client server communications that includes network element, network application and database. It shows communication between NMS application and the network element with help of communicator layer. It uses a client server approach with the help of different interface like X.25, TCP/IP and OSI.

Network management application includes

\subsection{Fault Management}

Fault management tools can help increase the reliability of the network by quickly identifying fault, and then help initiate the recovery process. It helps to identify the fault, isolate the cause of the fault, and then, if possible, correct the fault 


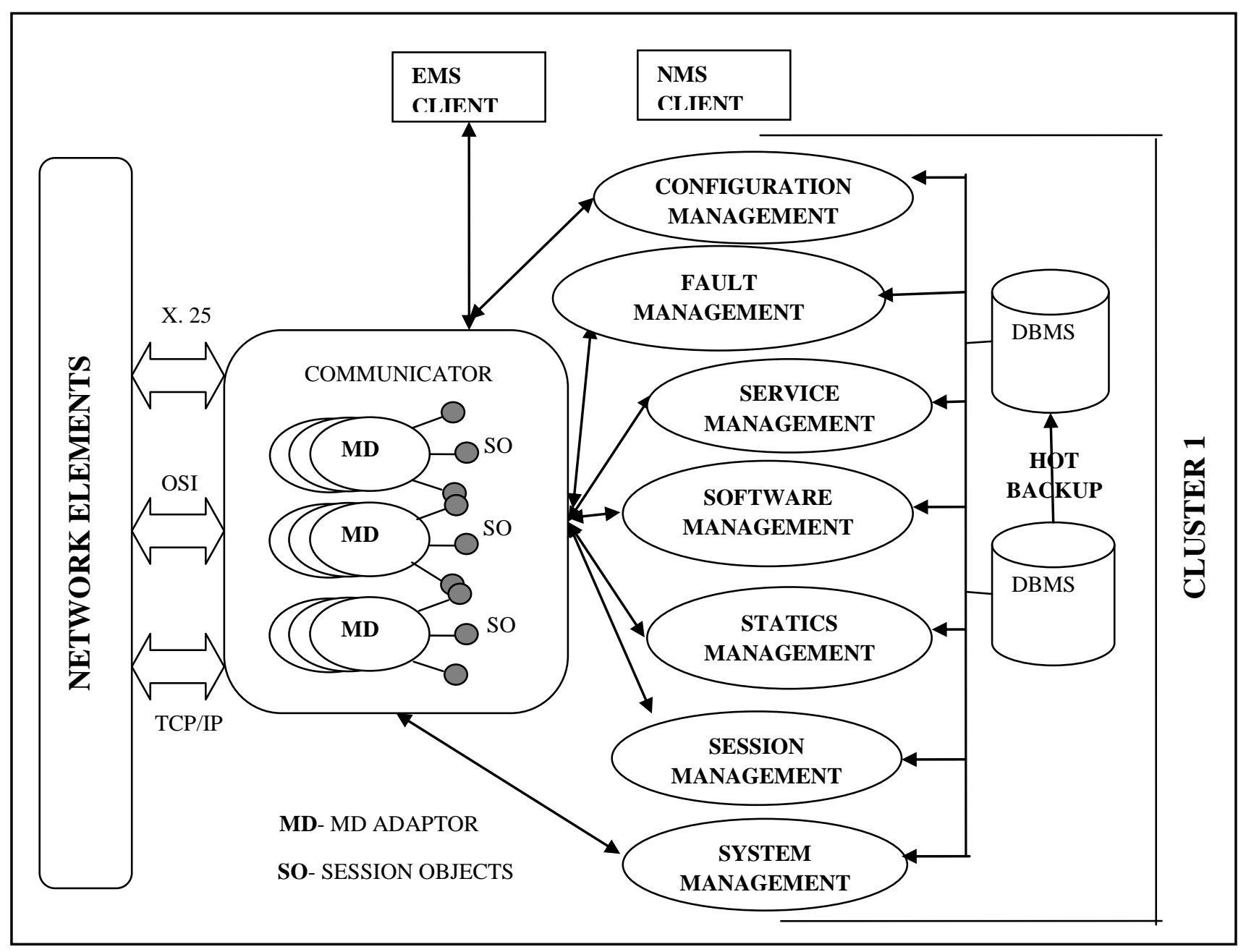

Fig 1: Client server based 3- tier model

\subsection{Configuration Management}

The process of configuration management involves identifying the network components and their connections. It helps to collect each device's configuration information, and define the relationship between network components. In order to perform these tasks, the network manager must knows, device configuration information, the network component control and information of topology of the network.

\subsection{Security Management}

It protects a network from unauthorized access and includes many sub-functions, such as the authorization of subscriber access, control of the distribution of cryptographic keying material, and the distribution and reporting of security related events.

\subsection{Accounting Management}

It allows the network manager to specify, log, and control user and device access to network resources. Usage quotas, usagebased charging, and the allocation of resource access privileges all fall under accounting management.

Network management and database management can be viewed as part of systems management or they may be viewed as co-equal parts of a total information system. The smallest logical system, necessary to provide the application level services is called cluster. Cluster provides the services which are used by application level. It also provides more hardware resources and distributes the component categories on multiple server hosts to introduce vertical scaling.

Cluster manages the whole or part of the managed devices network. A group of Network elements are managed by cluster. Distributed cluster provides a vertical scaling. By distributing the services on different server nodes and by adding more hardware resources, capacity and performance can be increased.

\section{DISTRIBUTED / DECENTRALIZATION OF NETWORK SYSTEM}

Low scalability and flexibility of client server introduces moving toward distributed or decentralization of network system [4]. In this kind of communicating applications with approaches like Management by delegation, Common Object Request Broker Architecture (CORBA)[5], implement reallife heterogeneous distributed applications. Web-based management, integrates all types of information systems into the internet or intranet environment[6]. Intelligent agents are introduced to communities of intelligent process to communicate for common task[7]. Active networks are framework which is used for transporting components to program and make them to execute code in order to achieve higher flexibility[8].

Distributed network management is inherently more robust without depending on continuous communications between the network management systems and network elements. 
Networks' traffic and processing load in the network. management systems can be both reduced by performing data processing closer to the network elements

Searches can be performed closer to the data, improving speed and efficiency. Scalability to large networks is improved. Processing capabilities for routers and switches have improved considerably and the popularizations of Java and CORBA have brought mobile code concepts closer to mainstream acceptance. New ways and designing should be implementing and introduced to handle the complexity of today's large networks. Code mobility is widely used by a new family of programming languages, generally called mobile code languages $[9,10]$.
Mobile codes are recognized as software that move to a heterogeneous networks environment, crosses different administration domains, and are automatically executed upon arrival at their destinations [11]. Mobile agent represents transportable or even active objects, they may move from one system to another to access remote resources or even meet other agents and cooperate with them. Mobile agents are an effective choice for many applications, for several reasons including improvements in latency and bandwidth of clientserver applications and reducing vulnerability to network disconnection. Although not all applications will need mobile agents, many other applications will find mobile agents the most effective implementation technique for all or part of their tasks.

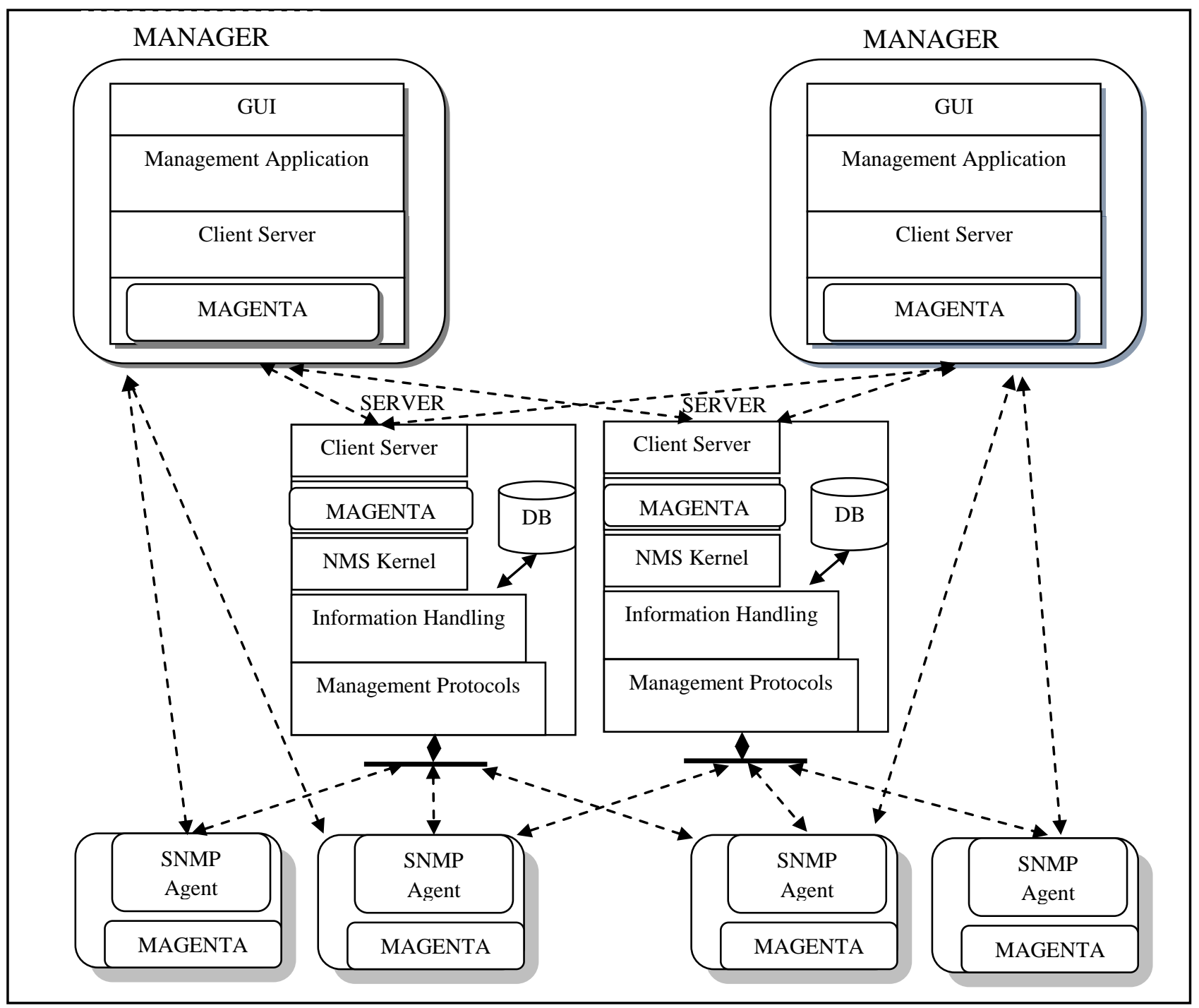

Fig 2: MAGENTA

In a broad sense, an agent is any program that acts on behalf of a (human) user.

A mobile agent then is a program which represents a user in a computer network, and is capable of migrating autonomously from node to node, to perform some computation on behalf of the user.

Benefits of Mobile Agent includes bandwidth conservation, reduction of latency reduction of completion time, asynchronous (disconnected) communications, load balancing, dynamic deployment

\section{MOBILE AGENT ENVIRONMENT FOR DISTRIBUTED APPLICATIONS (MAGENTA)}

Figure 2 shows mobile agents are furnished by MAGENTA (Mobile AGENT environment for distributed Applications) Mobile Network Manager (MNM) which is a location independent network manage[12].

The network managers in this architecture utilize clientserver technology and/or mobile agent technology as and 
when required depending on the functionality implemented and their location.

The architecture is distributed in nature as it provides for multiple managers acting simultaneously. It is rendered dynamic by the utilization of mobile agents for the implementation of network management functionalities
MAGENTA provides extensible and modifiable functionalities of the agents , fault - tolerance, dynamic adaptability to changes in the environment, flexibility in choice of location of agent code, simultaneous and fast execution of agents, true system independence along with access to system functions and the facility of remote execution[13]

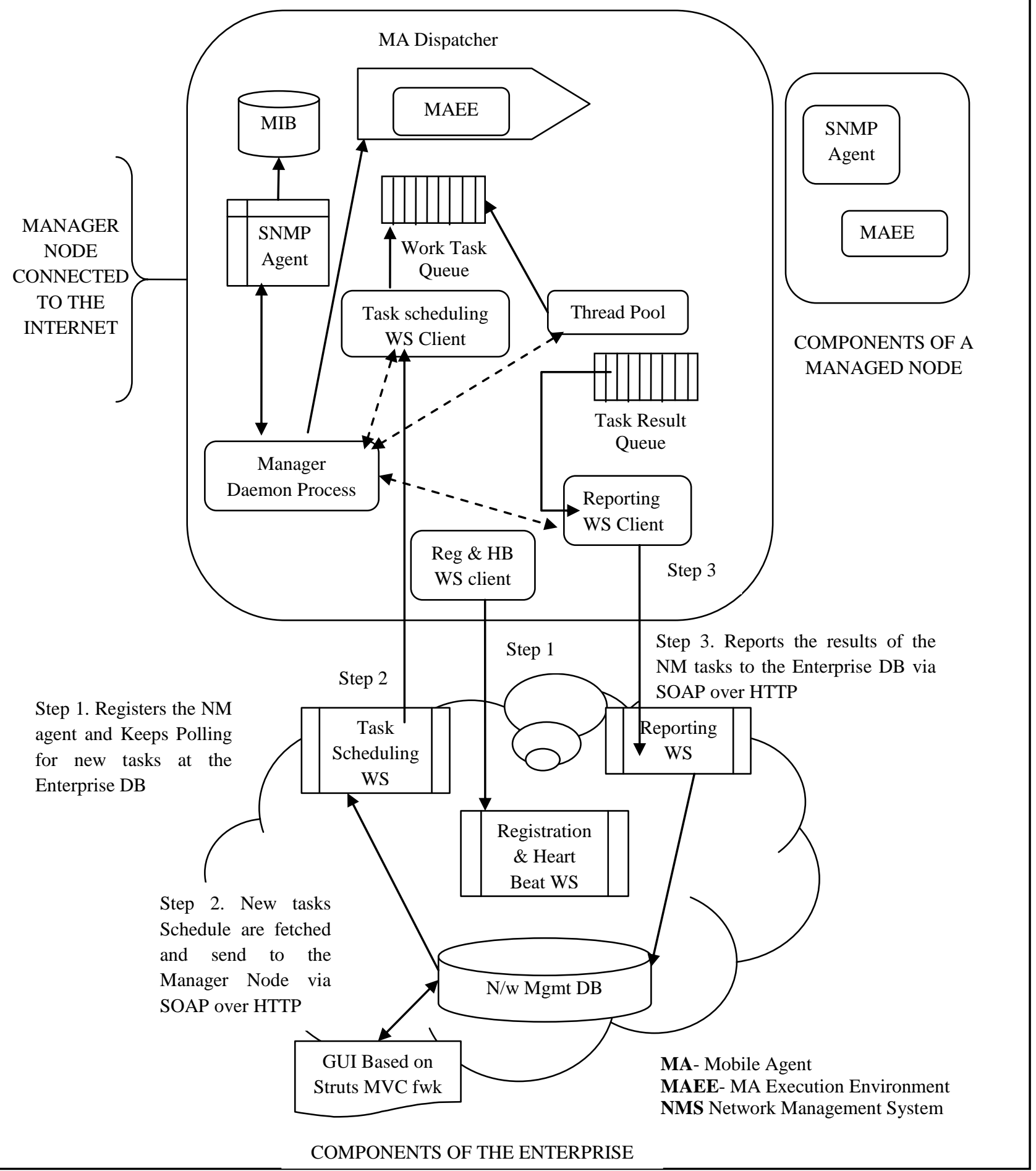

Fig 3: Netpatrol 


\section{NETPATROL}

The figure 3 shows Network Management that combined two seemingly divergent distributed computing technologies, namely, Web Services and Mobile Agents.

'Net Patrol' is a framework, in which the Mobile Agents 'patrols' / 'tours' the network by migrating to the network nodes scrutinizing its Memory, CPU, Bandwidth Utilizations etc. The network throughput is watched and prospective congestion segments are tracked and reported by overseeing the Packet Discard Rate at the NIC[14].

The name of this model is inspired by the name 'Net Doctor' which is used for the related work, done back in 1995, by Zapf et al [15], that uses MA and SNMP to manage networks. As shown in Figure 3, the framework broadly consists of three components namely: The Enterprise, The Manager Node, and The Managed Node

\section{Manager Node}

It has following components, a Daemon Process, MAEE (Mobile Agent Execution Environment), SNMP Agent, MIB (Management Information Base), 3 SOAP Based WS Clients, a pool of threads, result queue.

The network monitoring activity managed by mobile agents are

- Account Monitoring Mobile Agents ( ):

- Performance Monitoring Mobile Agents ( ):

- Root Cause Analysis Mobile Agents ( ):

The tasks to be executed are placed in the Work Queue of the Agent, which is implemented as a Thread-Pool Work Queue. This multi-threaded Agent execution ensures that many tasks can be concurrently executed[16].

\section{Enterprise}

'Net Patrol' uses a Service Oriented Enterprise, with the Web Services deployed on the Internet, thus enabling pervasive monitoring and management of the computer network. The 3 web services are Registration and Licensing Web Service, Task Scheduling Web Service and Result Reporting Web Service

\section{Managed Node}

It manages the following components: MAEE (Mobile Agent Execution Environment): 'Net Patrol' used Aglets Framework. SNMP Agent: 'Net Patrol' uses Advent Net SNMP API. MIB (Management Information Base): Maintains state of network parameters of itself. Set of library files developed to ease manager \& managed node communication

\section{HIERARCHICAL STATIC MIDDLE MANAGERS MODEL}

The scalability problem is more adequately addressed by deploying hierarchical models wherein NM tasks are delegated to MAs. They migrate to remote sub networks/domains where they act as local managers and takes over the responsibility of local devices from the central manager. These models suffer from automatic adaptation of management system to changing network configurations, i.e. mid level manager do not change the location where they execute. The model is shown in Figure 4[17].

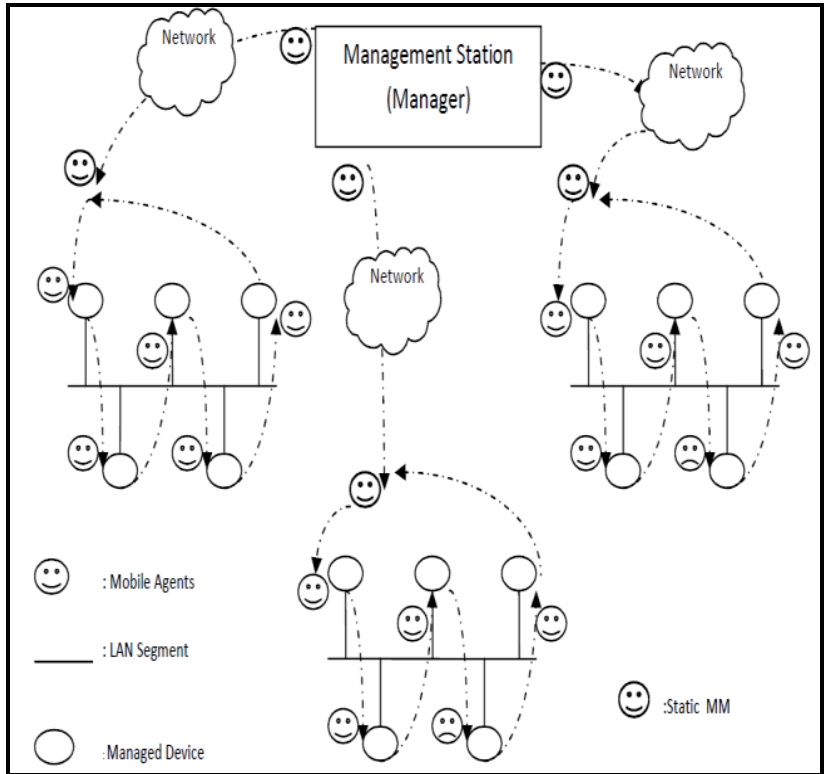

Fig 4: Hierarchical Static Middle Managers Model

\section{HIERARCHICAL MOBILE MIDDLE MANAGERS MODEL}

In search of more flexible solutions, a concept of Mobile Middle Manager (MDM), referring to a management component that operates at an intermediary level between the manager and the management end points, is introduced.

The mobility feature of the MDMs allows the management system to adapt dynamically to a changing network conditions. MDMs can be deployed to or removed from a given network segment in response to change in network traffic or move to a least loaded host to optimize local resource usage. The model is shown in Figure 5.

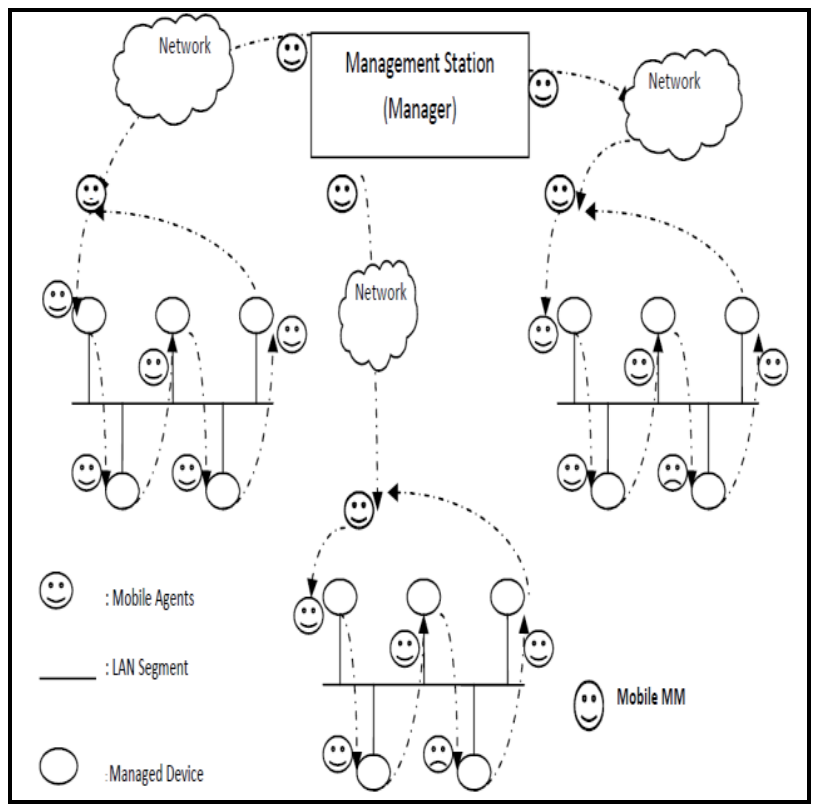

Fig 5: Hierarchical Mobile Middle Managers Model

These last two mobile agent-based network management models are discussed by Damianos Gavalas et al[18] in their research. 
Table 1. Comparative Analysis of various Network Management Model

\begin{tabular}{|c|c|c|c|c|c|}
\hline $\begin{array}{l}\text { Design } \\
\text { Parameters v/s } \\
\text { Models }\end{array}$ & Scalability & Load Balancing & Fault Tolerance & $\begin{array}{c}\text { Dynamic } \\
\text { Adaptation }\end{array}$ & $\begin{array}{c}\text { Integration } \\
\text { Of New } \\
\text { Services }\end{array}$ \\
\hline C/S Model & $\begin{array}{l}\text { Poor. The network gets } \\
\text { flooded with management } \\
\text { request/response messages. }\end{array}$ & $\begin{array}{l}\text { Low as every } \\
\text { management } \\
\text { decision is taken on } \\
\text { central manager. }\end{array}$ & $\begin{array}{l}\text { Once the link } \\
\text { between a sub } \\
\text { network and central } \\
\text { manager breaks, } \\
\text { that sub network } \\
\text { can't be managed }\end{array}$ & NO & NO \\
\hline $\begin{array}{l}\text { C/S BASED 3- } \\
\text { TIER MODEL }\end{array}$ & $\begin{array}{l}\text { Clusters are used to } \\
\text { improve the scalability, but } \\
\text { communicator uses C/S } \\
\text { approach to talk b/w NMS } \\
\& \text { NE. }\end{array}$ & $\begin{array}{l}\text { Low as every } \\
\text { communication is } \\
\text { done with help of } \\
\text { communicator }\end{array}$ & $\begin{array}{l}\text { If the link between } \\
\text { communicator \& } \\
\text { NE or NMS breaks, } \\
\text { difficult to handle }\end{array}$ & NO & YES \\
\hline MAGENTA & $\begin{array}{c}\text { Better, as location } \\
\text { independent manager } \\
\text { handles management task }\end{array}$ & $\begin{array}{c}\text { Uses mobile } \\
\text { network manager as } \\
\text { location } \\
\text { independent. }\end{array}$ & $\begin{array}{l}\text { Mobile Agents, } \\
\text { once dispatched, } \\
\text { can carry out the } \\
\text { management task } \\
\text { manage the } \\
\text { assigned even if the } \\
\text { link between them } \\
\text { and central } \\
\text { managers breaks. }\end{array}$ & YES & YES \\
\hline NETPATROL & $\begin{array}{l}\text { Combined two seemingly } \\
\text { divergent distributed } \\
\text { computing technologies, } \\
\text { namely, Web Services and } \\
\text { Mobile Agents }\end{array}$ & $\begin{array}{l}\text { Three SOAP Based } \\
\text { WS Clients: Vital } \\
\text { for Enterprise- } \\
\text { Agent SOAP } \\
\text { message exchange. }\end{array}$ & $\begin{array}{l}\text { Mobile Agents, } \\
\text { once dispatched, } \\
\text { can carry out the } \\
\text { management task } \\
\text { manage the } \\
\text { assigned even if the } \\
\text { link between them } \\
\text { and central } \\
\text { managers breaks }\end{array}$ & NO & YES \\
\hline H-SMM & $\begin{array}{l}\text { Better than the } \mathrm{C} / \mathrm{S}, 3 \text {-tier } \\
\mathrm{C} / \mathrm{S} \text { models as Static } \\
\text { Manager takes over the } \\
\text { management tasks from the } \\
\text { central manager. }\end{array}$ & $\begin{array}{l}\text { Higher as static } \\
\text { manager can relieve } \\
\text { the central manager } \\
\text { from mobile agents } \\
\text { generation and they } \\
\text { intern can utilize } \\
\text { local resources } \\
\text { more efficiently }\end{array}$ & $\begin{array}{l}\text { Static Managers can } \\
\text { manage their own } \\
\text { sub networks even } \\
\text { if the link between } \\
\text { them and central } \\
\text { managers breaks. }\end{array}$ & NO & YES \\
\hline H-MMМ & $\begin{array}{l}\text { Better than the } \mathrm{C} / \mathrm{S}, 3 \text {-tier } \\
\mathrm{C} / \mathrm{S} \text { based models as } \\
\text { Mobile Manager takes over } \\
\text { the management tasks from } \\
\text { the central manager. }\end{array}$ & $\begin{array}{l}\text { Highest as Mobile } \\
\text { middle manager can } \\
\text { re-locate itself as } \\
\text { the load on the host } \\
\text { (on which it is } \\
\text { running) increases. }\end{array}$ & $\begin{array}{c}\text { Mobile Managers } \\
\text { can manage their } \\
\text { own sub networks } \\
\text { even if the link } \\
\text { between them and } \\
\text { central managers } \\
\text { breaks. }\end{array}$ & YES & YES \\
\hline
\end{tabular}

\section{COMPARISON ANALYSIS OF C/S AND VARIOUS MA-BASED MANAGEMENT MODELS \\ All the MA-based management models enumerated in the} previous section and conventional client/server model (SNMP client/server model) are compared for five typical design parameters, i.e. scalability of management functions as the network grows, load balancing amongst participating entities, fault-tolerance of the management model, dynamic adaptation to the changing needs of network topology \& traffic patterns and easy integration of new management services in the management model. Table 1 summarizes the comparative study.

\section{CONCLUSION}

In client server SNMP uses get set request to communicate with the network element but Mobile agents offer an easy re- 
configurable, flexible and scalable solution to the management of today's complex telecommunication networks thereby reduces the number of necessary human interaction.

MAGENTA provides autonomous, reactive, proactive communicative mobile agents. As discussed, the independence and mobility of mobile agents reduce bandwidth overloading problems by moving a processing of the management data and decision making from centralized management stations to the managed devices thereby saving many repetitive request/response roundtrips and also address the problems created by intermittent or unreliable network connections between the network management stations and managed devices. Agents can easily work off-line and communicate their results when the application is back online. Moreover agents support parallel execution (load balancing) of large computation which can be easily divides among various computational resources. Thus decentralization solves many problems of centralized network system make the system efficient and increases the performance with the help of mobile agents.

\section{REFERENCES}

[1] Subramanian, M., Network Management Principles and Practice, Addison Wesley, 2000.

[2] W. Stallings. SNMP, SNMPv2 and CMIP: The practical guide to network management standards. Addisonwesley publication, 1994.

[3] Tripathi, A., Ahmed, T., Pathak, S., "Paradigms for Mobile Agent Based Active Monitoring Of Network Systems", IEEE, 2002.

[4] Shamila Makki "Next Generation Networks and Code Mobility".

[5] S. Vinoski, "CORBA: Integrating Diverse Applications Within Distribute Heterogeneous Environments", IEEE Communications Magazine, Vol. 35, No. 2, 1997.

[6] A. Liotta, G. Knight, and G. Pavlou, "Modeling Network and System Monitoring Over the Internet with Mobile Agents", IEEE, 1998.

[7] T. Magedanz, K. Rothermel, and S. Krause, "Intelligent Agents: An Emerging Technology for Next Generation Telecommunications”, INFOCOM '96, pp.24-28, 1996.
[8] D. Raz, B. Shavitt, "An Active Network Approach for Efficient Network Management", Proceedings of the $1^{\text {st }}$ International Working Conference on Active Networks (IWAN'99), LNCS Vol. 1653, pp. 220-231, 1999.

[9] J. E. White, "Telescript Technology: The Foundation for the Electronic Marketplace", General Magic White Paper, 1994.

[10] B. Joy et. al., "The Java Language Specification", 2nd ed. Reading, MA: Addison Wesley, 2000.

[11] A. Acharya, M. Ranganathan, and J. Saltz, "Dynamic linking for mobile programs, In Mobile Object Systems: Towards the Programmable Internet", pp. 245-262, Springer-Verlag, 1997.

[12] Akhil Sahai Christine Morin "Towards Distributed and Dynamic Network Management"

[13] Akhil sahai Christine morin "Enabling a mobile network manager through mobile agents"

[14] Mydhili K.Nair and V.Gopalakrishna "Applying Web Services With Mobile Agents For Computer Network Management'

[15] Zapf M, Herrmann K, Geihs K(1999),Decentralized SNMP Management with Mobile Agents, Proceedings of 6th Int. Symposium on Integrated Nwk Mgt, Boston, MA, USA, 24-28 May, 623-635

[16] Mydhili K Nair, Shishir M Kakaraddi, Keerthi M Ramnarayan, V Gopalakrishna(2009), Agent with Rule Engine:The 'Glue' for Web Service Oriented Computing Applied to NMS , Proceedings of IEEE Intl Conference on Services Computing(SCC 09),Bangalore, India, 528531

[17] Atul mishra \& A.k Sharma "Role of Agents in Distributed Network Management: A Review"

[18] Damianos Gavalas, Dominic Greenwood, Mohammed Ghanbari, Mike O'Mahony "Hierarchical Network Management: A Scalable and Dynamic Mobile AgentBased Approach" 